\title{
BMJ Open Stepped-wedge cluster randomised controlled trial to assess the effectiveness of an electronic medication management system to reduce medication errors, adverse drug events and average length of stay at two paediatric hospitals: a study protocol
}

\author{
J I Westbrook, ${ }^{1}$ L Li, ${ }^{1}$ M Z Raban, ${ }^{1}$ M T Baysari, ${ }^{1}$ V Mumford, ${ }^{1}$ M Prgomet, ${ }^{1}$ \\ A Georgiou, ${ }^{1}$ T Kim, ${ }^{1} \mathrm{R}$ Lake, ${ }^{1} \mathrm{C}$ McCullagh, ${ }^{2}$ L Dalla-Pozza, ${ }^{2} \mathrm{~J}$ Karnon, ${ }^{3}$ \\ T A O'Brien, ${ }^{2} \mathrm{G}$ Ambler, ${ }^{4} \mathrm{R}$ Day, ${ }^{5} \mathrm{C}$ T Cowell, ${ }^{2} \mathrm{M}$ Gazarian, ${ }^{5} \mathrm{R}$ Worthington, ${ }^{2}$ \\ C U Lehmann, ${ }^{6}$ L White, ${ }^{7}$ D Barbaric, ${ }^{2}$ A Gardo, ${ }^{2}$ M Kelly, ${ }^{7}$ P Kennedy ${ }^{8}$
}

To cite: Westbrook JI, Li L, Raban MZ, et al. Steppedwedge cluster randomised controlled trial to assess the effectiveness of an electronic medication management system to reduce medication errors, adverse drug events and average length of stay at two paediatric hospitals: a study protocol. BMJ Open 2016;6:e011811.

doi:10.1136/bmjopen-2016011811

- Prepublication history and additional material is available. To view please visit the journal (http://dx.doi.org/ 10.1136/bmjopen-2016011811).

Received 7 March 2016 Revised 18 July 2016 Accepted 28 September 2016

\section{CrossMark}

For numbered affiliations see end of article.

Correspondence to Professor JI Westbrook; johanna.westbrook@mq. edu.au

\section{ABSTRACT}

Introduction: Medication errors are the most frequent cause of preventable harm in hospitals. Medication management in paediatric patients is particularly complex and consequently potential for harms are greater than in adults. Electronic medication management (eMM) systems are heralded as a highly effective intervention to reduce adverse drug events (ADEs), yet internationally evidence of their effectiveness in paediatric populations is limited. This study will assess the effectiveness of an eMM system to reduce medication errors, ADEs and length of stay (LOS). The study will also investigate system impact on clinical work processes.

Methods and analysis: A stepped-wedge cluster randomised controlled trial (SWCRCT) will measure changes pre-eMM and post-eMM system implementation in prescribing and medication administration error (MAE) rates, potential and actual ADEs, and average LOS. In stage 1, 8 wards within the first paediatric hospital will be randomised to receive the eMM system 1 week apart. In stage 2, the second paediatric hospital will randomise implementation of a modified eMM and outcomes will be assessed.

Prescribing errors will be identified through record reviews, and MAEs through direct observation of nurses and record reviews. Actual and potential severity will be assigned. Outcomes will be assessed at the patient-level using mixed models, taking into account correlation of admissions within wards and multiple admissions for the same patient, with adjustment for potential confounders. Interviews and direct observation of clinicians will investigate the effects of the system on workflow. Data from site 1 will be used to develop improvements in the eMM and implemented at site 2, where the SWCRCT design will be repeated (stage 2).
Strengths and limitations of this study

- The stepped-wedge cluster randomised controlled trial (SWCRCT) study design is the closest to the gold standard of a randomised controlled trial, and has rarely been applied to study the effects of information technology in healthcare.

- Few previous studies have investigated the impact of electronic systems on medication administration error rates. We present a novel approach to conduct direct observation of this process using the Precise Observation System for Safe Use of Medicines (POSSUM). POSSUM allows observers to quickly and accurately record drug information, for example, name, strength, compliance with procedures, as well as the number and length of interruptions and multitasking.

- We will assess potential harm from medication errors identified and importantly also measure actual harm to children.

- Direct, close observation lends itself to the 'Hawthorne effect' whereby participants may seek to 'improve' their performance. This may result in an underestimation of the 'true' medication administration error rate.

- This study will not evaluate adverse drug events occurring postdischarge.

Ethics and dissemination: The research has been approved by the Human Research Ethics Committee of the Sydney Children's Hospitals Network and Macquarie University. Results will be reported through academic journals and seminar and conference presentations. Trial registration number: Australian New Zealand Clinical Trials Registry (ANZCTR) 370325. 


\section{INTRODUCTION}

Prescribing, administering and monitoring medicines in children is highly complex. Compared with adults, medication errors in children are three times more likely to result in harm. ${ }^{1}$ Paediatric patients are at particular risk of certain errors, such as 10 -fold dosing errors facilitated by mistakes in dose calculation, poor documentation of decimal points and confusion with the use of zeros. Some errors have led to death. ${ }^{2-4}$ The complex medication decision process in children often requires information about age, weight, dosing ranges and off-label use of medicines. ${ }^{2}$ Systematic reviews show errors occur in $5-27 \%$ of all medication orders for children. ${ }^{5-7}$ Prescribing errors account for $3-37 \%$ of errors, dispensing $5-58 \%$, administration $72-75 \%$ and documentation $17-21 \%$, although accuracy of estimates is affected by the great variability in definitions and measurement methods used. ${ }^{6}$ There is much less research about the harms associated with medicine use. One Australian study found $19.2 \%$ of paediatric inpatients experienced adverse drug events (ADEs) and estimated $12.3 \%$ were potential ADEs, $7.0 \%$ were actual ADEs and $3.6 \%$ were preventable. $^{8}$

The frequency and severity of medication administration errors (MAEs) in Australian adult hospitals is a cause for concern. ${ }^{9}{ }^{10}$ In 4271 medications administered to 720 patients, $74.4 \%$ were found to have at least one procedural failure (eg, failure to check a patient's identification). ${ }^{9}{ }^{10}$ One in four had a clinical error (eg, wrong dose). Of intravenous drug administrations, $70 \%$ had one clinical error, of which $25.5 \%$ were judged to be serious and likely to cause permanent harm. ${ }^{9}$ MAEs among children are rarely studied. ${ }^{6}{ }^{11}$ A major barrier to MAE research is the methodological challenges. MAEs cannot be accurately detected from retrospective record reviews; they require direct observation of nurses administering medications to patients.

\section{Can information technology reduce medication errors?}

Electronic medication management (eMM) systems incorporate software which provides users with the ability to prescribe, monitor and administer medications to patients. These systems also provide the capacity to incorporate decision support tools such as alerts for drug-drug interactions. ${ }^{12}$ eMM is usually integrated into a hospital's clinical information system (computerised provider order entry system). These systems are expected to reduce medication errors and ADEs significantly as a result of improved legibility of medication orders, complete and legally compliant documentation, and through both the active and passive decision support tools embedded in them. However, rigorous evidence demonstrating these effects is limited. ${ }^{7}$ A systematic review identified eight studies of eMM effectiveness among paediatric patients. Meta-analysis showed a significant reduction in prescribing error risk (RR 0.08; $95 \%$ CI 0.01 to 0.77 ) across the three included studies, but not in ADEs or mortality. ${ }^{13}$ There are no Australian studies of eMM system use in a paediatric setting. Previous studies have often relied on incident reports to measure error rates, which are generally unreliable. ${ }^{14}$ Only one study ${ }^{15}$ of paediatric inpatients has used a control group to assess eMM effectiveness. Internationally there is currently insufficient evidence to demonstrate clinical benefit from eMM in paediatric patients. This view was confirmed by a policy review for the American Academy of Pediatrics ${ }^{16}$ which called for the demonstrable enhancement of eMM systems to better meet the specific needs of paediatrics to ensure their safety and effectiveness. Further, existing eMM systems used in paediatrics have been found lacking in functionalities required for safe prescribing, dispensing and administration of medications. ${ }^{17} 18$

In 2005 , Han et $a l^{19}$ reported a significant increase in the mortality rate among critical care children at a US paediatric hospital following introduction of a commercial eMM system. The rapid implementation process and limited attention to the significant workflow redesign required were considered as major factors in this outcome. Subsequent studies ${ }^{20}$ have shown no increase in mortality rates. However, the Han $e t a l^{19}$ study caused considerable alarm and served to demonstrate the substantial dangers of poor implementation and the importance of monitoring outcomes following system implementation and responding to the problems identified. ${ }^{21}$

eMM use in adult hospitals, while highly effective at reducing medication errors, ${ }^{22}$ also introduced new 'system-related' errors. An investigation of 1164 prescribing errors post-eMM in two adult Australian hospitals found $42.4 \%$ were facilitated by the system (78 per 100 admissions). ${ }^{23}$ The most frequent mechanism was incorrect selection from a drop-down menu. Results from that study were used to instigate changes to eMM software and to inform changes to user training programmes. The study was one of the first to quantify the rate of 'system-related' errors and produced an innovative dual classification for categorising both the manifestations and mechanisms of these system-related errors. ${ }^{23}$ The nature and magnitude of such potential new risks have rarely been studied in paediatrics.

\section{Why evaluate eHealth systems}

eHealth systems are having an increasing impact on the delivery of healthcare services, yet, despite their widespread effects and vast cost, they are rarely subject to rigorous research. ${ }^{24}$ This limited evidence base significantly hinders improvements and innovation in the design, implementation and use of health information technology (IT) systems. Internationally the need for IT evaluation studies to employ more robust designs and sophisticated analyses is well recognised..$^{25}$ Excessive reliance has been placed on uncontrolled before-and-after and qualitative studies. Evidence of eHealth system effectiveness and safety is crucial to facilitate policymakers' and healthcare organisations' informed 
decisions about investments and prioritisation of health IT systems.

This project presents an innovative and comprehensive programme to assess eMM system's effectiveness in reducing medication errors, ADEs and length of stay (LOS) and rapidly deploying new knowledge into practice for subsequent implementation.

The project aims are:

Aim 1: to quantify the safety and effectiveness of an eMM system to reduce medication errors, ADEs and average LOS among paediatric patients using a stepped-wedge cluster randomised controlled trial (SWCRCT) design;

Aim 2: to assess the effects of the eMM on clinicians' workflow and efficiency; and

Aim 3: to assess the extent to which feedback of study results and subsequent modifications to the eMM design and associated work practices can improve eMM effectiveness in reducing medication errors at a subsequent implementation tested via a second SWCRCT.

\section{METHODS AND ANALYSIS \\ Research plan}

Aim 1

Quantify the safety and effectiveness of an eMM system to reduce medication errors (potential and actual ADEs), and average LOS among paediatric patients.

\section{Study design and setting}

We will conduct a SWCRCT to measure changes in prescribing and MAEs which result in potential and actual ADEs, along with changes in average LOS, pre-eMM and post-eMM. Cluster randomised controlled trials (CRCT) are ideally suited to test interventions where individual patient randomisation is not possible. CRCTs commonly use a parallel group design, in which the clusters are randomised to either the intervention or the control arm of the study. It is often regarded as unethical to withhold an intervention from a proportion of participants if it is believed that the intervention will do more good than harm. The SWCRCT design, where the intervention is delivered sequentially to all trial clusters over a number of time periods, is an alternative to the traditional parallel groups design. The order in which the clusters (wards) receive the intervention is randomised, and by the end of the study all clusters will have adopted the intervention. ${ }^{26}$ The steps represent the predetermined periods when data relating to each of the clusters will be collected. This design is the closest to the gold standard of an RCT, when such a design is not possible. The stepped-wedge design offers particular strengths in allowing the modelling of the effect of time on the effectiveness of the intervention. In stage 1 a SWCRCT will be conducted at site 1 , and in stage 2 a SWCRCT will be conducted at site 2 .

The study setting is the Sydney Children's Hospitals Network which incorporates the two major urban tertiary referral paediatric hospitals in Sydney, Australia, The Children's Hospital at Westmead (CHW; site 1) and Sydney Children's Hospital, Randwick (SCH; site 2). The network provides care for 51000 inpatient admissions, 92000 emergency department presentations and over 1000000 outpatient services events each year. Both sites are acute paediatric tertiary hospitals with emergency departments, outpatient and home services. During the study period, the eMM will not be available in the intensive care units (ICUs), theatres or outpatients. Site 1 accommodates 310 beds and site 2 has 180 beds. Both hospitals provide a complex and comprehensive range of services caring for seriously ill and injured children and adolescents across the state of New South Wales and beyond.

\section{The eMM intervention}

At baseline medication orders are written on paper medication charts and details of medications administered are written on the same charts. The eMM clinical module will be an addition to the hospitals' existing commercial electronic clinical information system (Cerner Corporation). Both hospital sites use the same commercial clinical system; however, the software can be customised to meet each hospital's individual requirements. Based on the results from our first site and applying our action research methodology, we will provide advice to the second implementation site on optimal customisation of the eMM (and associated processes) for their site. The eMM allows electronic prescribing, recording of drug dispensing, drug administration and medication reconciliation and monitoring processes (see online supplementary material file for screenshots from the eMM). The system allows for the ordering and administration of all oral, and intrvenous medications and fluids, but excludes anaesthesia medications. The eMM contains both passive and active decision support in the form of links to guidelines, policies, protocols, order sets, order sentences, safety alerts (eg, drug-drug interactions, dose range checks) and dosage calculators. During the course of the study, the eMM system will be accessible via any computer in the hospital allocated for inpatient clinical care, but will not be available for patients in the ICUs, theatres or outpatients. The system will be predominantly accessed in hospital wards and in the hospital pharmacy. Both fixed and mobile computing devices are available to staff using the system.

Medication reconciliation on admission and at discharge will be performed using the eMM system when implemented. On admission, medication histories are taken and converted to inpatient orders. While the patient is in hospital any new medication orders will be created within the eMM system. On discharge, a discharge medication reconciliation occurs and orders are converted to paper prescriptions for the patient. Patients then have their prescriptions filled at community pharmacies. 


\section{Randomisation}

In stage 1 , eight wards within site 1 will be randomised to receive the eMM system. Randomisation will be conducted by a person blinded to ward identity. At baseline, all eight wards are in the control period (figure 1). At step 1 , the eMM system will be implemented in the first ward. The eMM system will then be implemented to a new ward in sequence weekly. By the end of step 8, all wards will have the eMM system at site 1 .

\section{Medication error and ADE definitions}

Medication errors are defined as any error in the prescribing, supply, preparation, administration or monitoring of a medication, regardless of whether such errors lead to adverse consequences. In this study, we will not measure dispensing errors. ADEs are defined as harm or injury as a consequence of the use or non-use of medicines. ${ }^{8}$ Medication errors may result in actual ADEs or potential ADEs. For example, a medication error may occur but is intercepted prior to administration thus preventing harm to the patient.

In this study, we will be seeking to identify medication errors, and to determine those that resulted in harm (actual ADEs) or potential harm (potential ADEs). Figure 2 illustrates the medication error and ADE classification processes for this study.

\section{Data collection}

Medication error data collection occurs at baseline ( 1 week period) and each step (ie, in every subsequent week as eMM implementation occurs and for 2 additional weeks after full implementation). For the primary study objective of determining eMM effectiveness to reduce medication errors and ADEs, we will collect data at 11 points on all wards (baseline and at each step including 2 weeks after full implementation, figure 1). This will allow us to measure changes pre-eMM and post-eMM system introduction in: (1) prescribing error rates per order and per admission by type and severity (potential and actual ADEs); (2) MAEs per order and per admission by type and severity (potential and actual ADEs). For the secondary outcome of changes in LOS, we will obtain data for a further 21 steps in the follow-up period to provide greater statistical power. As these are routine administrative data, no additional data collection is required. Data on ADEs occurring postdischarge is not within the scope of this study.
Prescribing error and ADE detection: A review of medication charts at baseline and each step will be conducted complying with a standard error protocol. ${ }^{8-10} 22$ This protocol will be extended to develop methods for determining the rate at which errors are detected and intercepted by staff, actions taken and any harm experienced.

MAE and ADE detection: For the MAE study, data will be collected using direct observation. Nurses will be observed preparing and administering medications. In our previous studies using this approach in adult hospitals, ${ }^{10}$ over $80 \%$ of nurses consented to participate and we expect similar rates for this study. Direct observations will be supported by an innovative data collection tool, the Precise Observation System for Safe Use of Medicines (POSSUM, figure 3)..$^{10} 27$ The POSSUM tool allows observers to quickly and accurately record drug information, for example, name, strength and dose. The POSSUM tool also allows collection of the number and length of interruptions experienced and multitasking (eg, answering a question while also selecting medicines). Nurses' compliance with core procedures, such as checking a patient's identification, will also be recorded. Comparing observational data with patients' medical records (via retrospective audit) will enable identification of the number, types and severity of MAEs.

Observers will have a preallocated observation period to ensure coverage across the day and the week. ${ }^{9}{ }^{10}$ Observers will follow a 'serious error' protocol, that is, they must intervene if they witness an administration that is potentially dangerous to the patient. Observers will not have access to patients' medication charts and will record only what they observe. Thus, most MAEs will not be identifiable until chart review. Past inter-rater reliability tests showed $\kappa$ scores from 0.94 to 0.96 following training in the use of POSSUM. ${ }^{9} 10$

Direct, close observation lends itself to the 'Hawthorne effect' whereby participants may seek to 'improve' their performance. If nurses change their practices, and are more careful when observed, this will lead to an underestimation of the 'true' MAE rate. This bias would be present both pre-eMM and post-eMM. Our prior research suggests the likelihood of sustained change on busy wards is low. ${ }^{28} 29$

Prescribing error, MAE and ADE classifications: Prescribing errors and MAEs will be classified into: (1) procedural errors and (2) clinical errors using previously

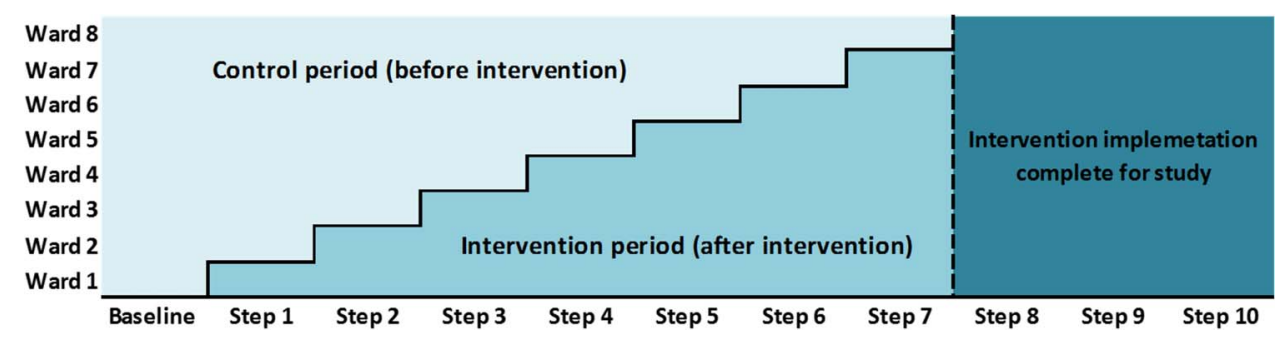

Figure 1 Schematic of stepped-wedge cluster randomised controlled trial study design. 


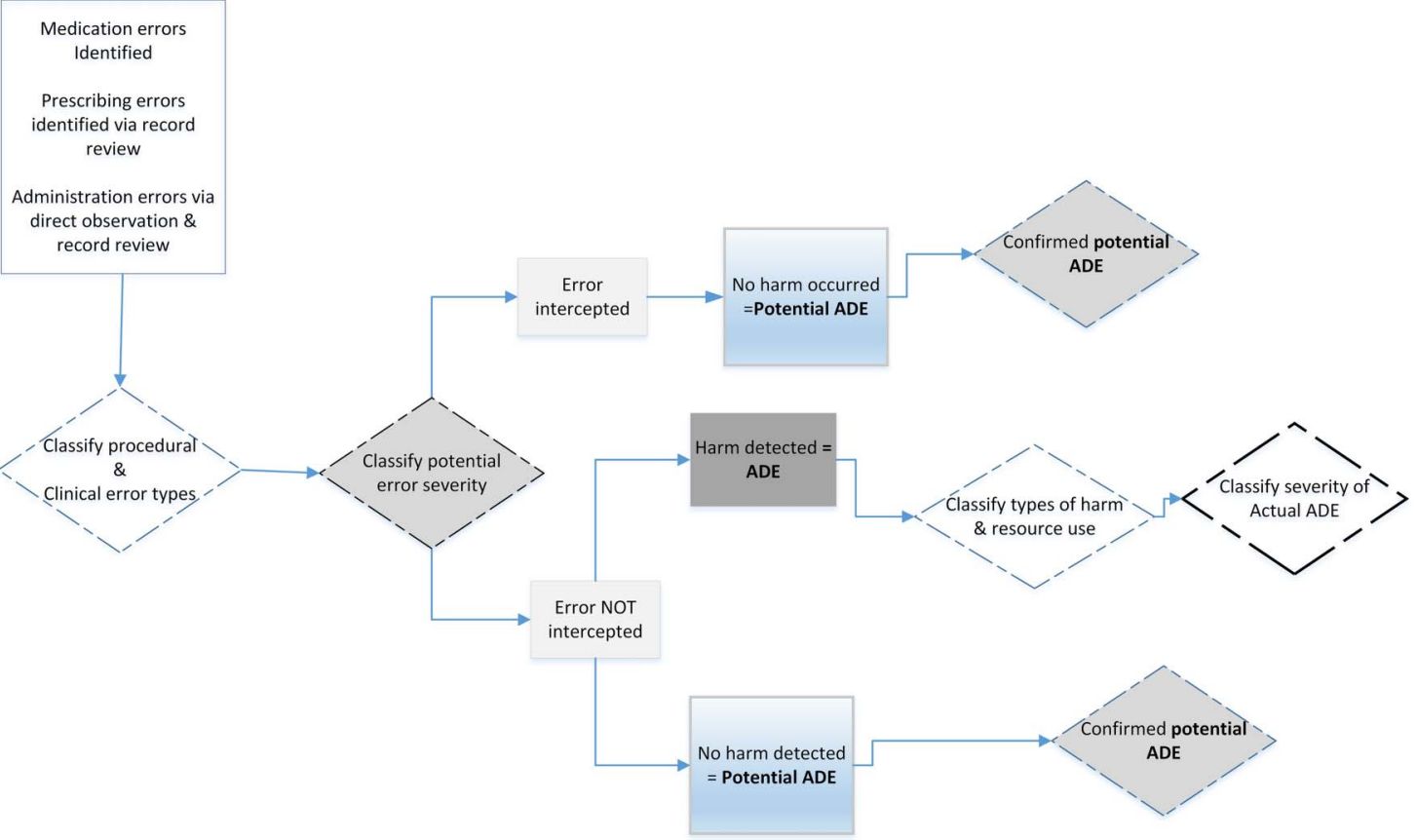

Figure 2 Medication error, ADE and harm identification and classification process. ADE, adverse drug event.

applied classifications. ${ }^{10} 22$ Procedural errors include, for prescribing orders, illegible orders, illegal orders (in which an aspect of the prescription does not comply with hospital policy, with law, or with the State Department of Health policies) and incomplete orders. Medication administration procedural errors include, for example, failure to follow the correct patient identification process prior to drug administration, and correct conduct of medication double-checking requirements (many drugs within paediatric hospitals require two nurses to independently check aspects of the drug preparation and administration process). Clinical errors include wrong dose, wrong drug, wrong route and wrong strength errors.

Once an error has been identified, a rating of the potential severity of that error will be made, based on the National Coordinating Council for Medication Error Reporting and Prevention (NCC-MERP) rating scale for adverse event outcomes. ${ }^{30}$ Subsequently, records will be reviewed for the evidence of error detection and interception, and for any actual harm to the patient. Thus, medication errors which occur will receive both a 'potential' harm rating and an 'actual harm rating' (figure 2). As most previous medication error studies do not assess actual harm, this double classification process will allow us to compare our findings with previous studies, as well as allow an assessment of the accuracy of such approaches compared with estimating the actual harm from medication errors.

Evidence of harm as a consequence of a medication error will be identified through a comprehensive review of patients' medical records. This clinical review process will be assisted by the provision of specific harm identification guides for reviewers which will identify, for specific drugs and error types, the types of evidence which would suggest harm had occurred following the medication error. Figure 4 presents an example of one of the harm identification guides to be used.

Experienced clinicians will abstract data from medical records using a structured data collection form and the harm identification guides. A multidisciplinary clinical review panel will reassess a minimum $5 \%$ sample of the records and will also review any records which reviewers identify as particularly complex. Panel members will be blind to the location, and whether data were generated pre-eMM or post-eMM. Panel members will not know the ward order of rollout and specific dates when each ward became an intervention ward with the stepped-wedge design and therefore blinding of predata and postdata will be possible. Actual and potential severity will be assigned using the NCC-MERP scale for adverse event outcomes $^{30}$ and the 5-point Severity Assessment Code (SAC) Scale, ${ }^{31}$ as used in our past research. ${ }^{9} 1022$ This will allow comparison with a greater number of previous studies.

System-related errors: We will apply our two-dimensional classification, modified to incorporate recent recommendations in this area, ${ }^{21}$ to assess whether medication errors post-eMM were facilitated by eMM design, that is, are 'system-related'. This process identifies the manifestation (eg, wrong dose) and mechanisms (eg, incorrect menu selection). These results will be used to provide recommendations about IT design and user training. ${ }^{23}$ Any changes to the eMM design features, training or work processes during the study will be documented.

\section{Sample sizes and analyses}

Sample size calculations have taken into account the estimated between-cluster variance, that is, between wards 
Figure 3 POSSUM tool for data collection during the direct observational study of medication administration. POSSUM, Precise Observation System for Safe Use of Medicines.

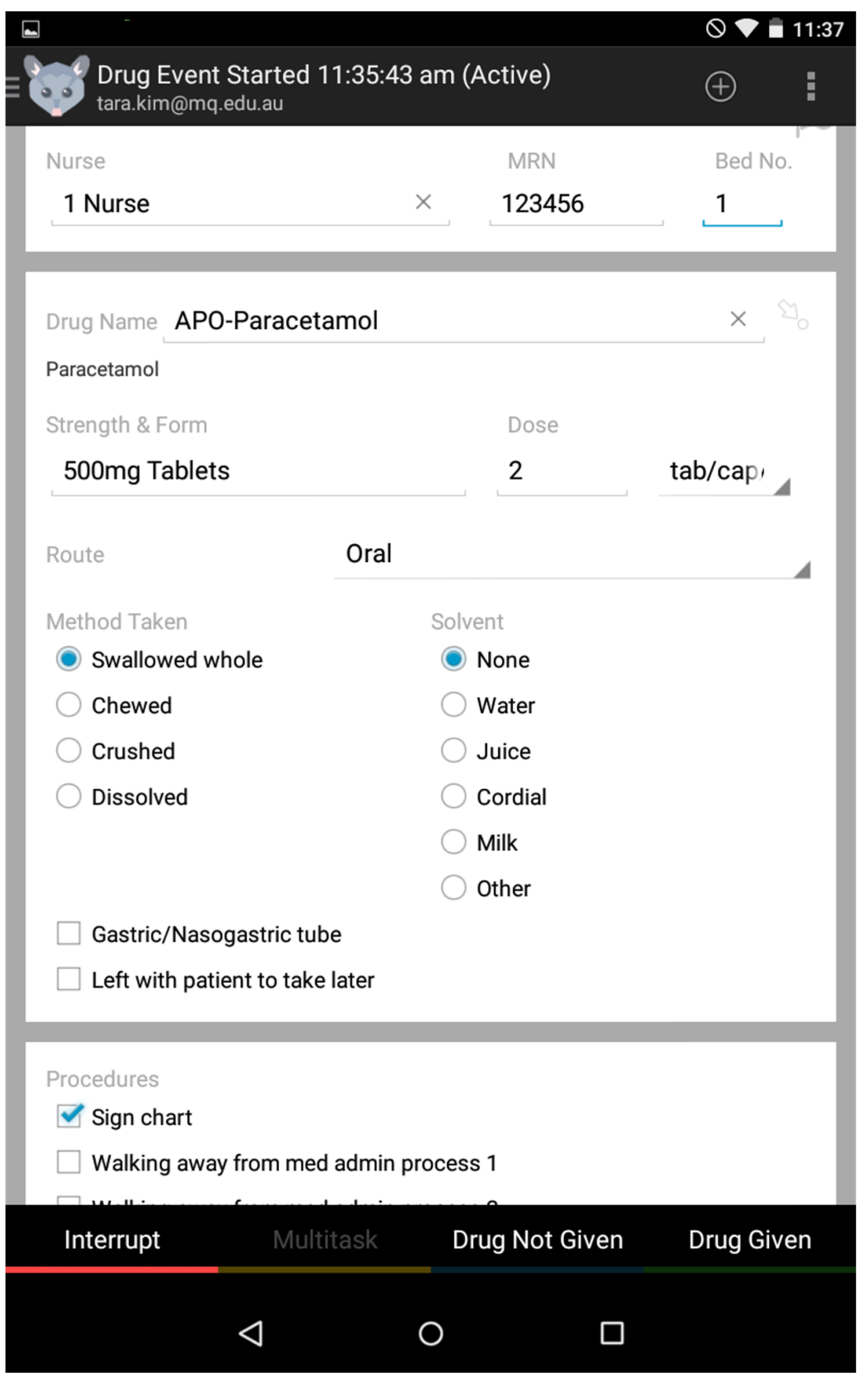

variance, and the design effect associated with the stepped-wedge design. ${ }^{32}$ Calculations were based on our previous studies in adult hospitals and hospital data from the paediatric sites. Each ward has on average 14 admissions per week with an average LOS 3.78 days $(\mathrm{SD}=7.39)$ with seven medications per admission.

Prescribing errors: Based on our previous studies ${ }^{22}$ the expected reduction in overall prescribing error rate is $60 \%$, from 4.06 errors per admission $(\mathrm{SD}=5.27)$ to 1.62 $(\mathrm{SD}=2.87)$ with an estimated intraclass correlation coefficient (ICC) of 0.06 (table 1). The number of wards required to detect a $60 \%$ change for two-sided tests ( $80 \%$ power; $\alpha<5 \%$ ) is one, with 10 data collection steps after baseline. For ADEs, the required number of wards is seven to detect a $60 \%$ reduction (table 1 ). To be conservative and provide greater power, we will collect data on all eight wards allowing detection of a minimum change of $20 \%$ for overall errors and $42 \%$ for ADEs. At each step records for 112 patient admissions will be reviewed, totalling 1232 across the study.

MAEs: Based on our previous studies ${ }^{9}{ }^{10}$ we expect the overall MAE rate per administration to fall by $27 \%$, from 
Figure 4 Example of harm assessment guide for paediatric opioid errors, to be used during medical record review following identification of an opioid prescribing error. BP, blood pressure; ICU, intensive care unit.

\begin{tabular}{|c|c|c|}
\hline $\begin{array}{l}\text { Medication } \\
\text { Error results } \\
\text { in: }\end{array}$ & $\begin{array}{l}\text { Higher than recommended/intended } \\
\text { dose or concentration of Opioid. }\end{array}$ & $\begin{array}{l}\text { Lower than recommended/intended } \\
\text { dose or concentration of Opioid. }\end{array}$ \\
\hline Symptoms & $\begin{array}{l}\text { Drowsiness, nausea, vomiting, } \\
\text { agitation/restlessness, constipation*, and } \\
\text { itch* }\end{array}$ & $\begin{array}{l}\text { Complaints of pain, agitation/restlessness } \\
\text { (due to pain) }\end{array}$ \\
\hline Signs & $\begin{array}{l}\text { Pinpoint pupils, lower level of } \\
\text { consciousness, respiratory rate below age } \\
\text { related normal range, sedation score }>3 \text {, } \\
\text { opioid related cardio-respiratory arrest, } \\
\text { unexpected oxygen desaturation requiring } \\
\text { supplemental oxygen }\end{array}$ & $\begin{array}{l}\text { Pulse and BP above normal range, pain } \\
\text { scores noted and increasing. Pain scores }>6 \\
\text { for two or more hours }\end{array}$ \\
\hline Medications & $\begin{array}{l}\text { Naloxone, abrupt cessation of opioid, } \\
\text { commencement of laxatives*, inadvertent } \\
\text { prescription of more than one opioid. }\end{array}$ & Additional doses of analgesic/s \\
\hline Tests & $\begin{array}{l}\text { Blood gases - high } \mathrm{CO} 2, \mathrm{O} 2 \text { saturation } \\
<95 \%\end{array}$ & \\
\hline Actions & $\begin{array}{l}\text { Increased monitoring and increased } \\
\text { clinical observations, rapid response call, } \\
\text { supplemental oxygen }\end{array}$ & Pain or anaesthetic team call back. \\
\hline Care record & $\begin{array}{l}\text { Increased level of care (e.g. transfer to } \\
\text { ICU), family notified, incident report } \\
\text { filed, discharge delayed }\end{array}$ & Opioid withdrawal score $>10$ \\
\hline References & $\begin{array}{l}\text { Niesters M, Overdyk F, Smith T, et al. } \\
\text { Opioid-induced respiratory depression in } \\
\text { paediatrics: a review of case reports. } B r J \\
\text { Anaesth } 2013 ; 110(2): 175-82 \text {. } \\
\text { Sharek PJ, McClead RE, Jr., Taketomo C, } \\
\text { et al. An intervention to decrease narcotic- } \\
\text { related adverse drug events in children's } \\
\text { hospitals. Pediatrics 2008;122(4):e861- } \\
6 .=\end{array}$ & \\
\hline
\end{tabular}

$0.37(\mathrm{SD}=0.65)$ to $0.27(\mathrm{SD}=0.52)$ with an estimated $\mathrm{ICC}$ of 0.03 (table 1). The required number of wards (twosided test; $80 \%$ power; $\alpha<5 \%$ ) is seven, with 10 steps after baseline. For ADEs, the required number of wards is six. We will collect data using all eight wards which will allow detection of a minimum $20 \%$ change overall and $48 \%$ for ADEs. At each step we will observe at least 240 medication administrations, totalling 2640 across the study.

LOS: There are very limited data on the impact of eMM systems on LOS at ward level. A study in an ICU showed a $23 \%$ reduction in LOS post-eMM system. ${ }^{33}$ To detect a $23 \%$ reduction in LOS, that is, from 3.78 $(\mathrm{SD}=7.39)$ to 2.92 days, with eight wards, will require (routinely collected LOS) data in a total of 31 steps for a two-sided test with $80 \%$ power $\alpha<5 \%$.

\section{Data analyses}

Medication error rates per order, stratified by error type, study step and ward will be calculated. For each outcome of interest, data collected across all measurement periods and all study steps will be used in the analyses comparing intervention status (pre-eMM vs post-eMM). Analyses will apply the intention-to-treat principle. Patient data will be analysed according to the status of the wards (ie, pre-eMM or post-eMM) where patients were admitted. Outcomes will be assessed at the patient level using mixed models, taking into account correlation of patient admissions within wards (clusters) and multiple admissions for the same patient, with adjustment for potential confounding factors. For the MAE analyses, we will adjust for contextual factors including interruptions, multitasking, nurse age, gender and adherence to policies. For LOS analysis we will adjust for patient characteristics, such as major diagnoses, comorbidity, age and gender. The mixed models will incorporate fixed terms for ward intervention status, measurement time steps (including baseline) and other confounders. The analyses will include multiple time points pre-eMM and post-eMM implementation. The study design will allow us to determine temporal changes in system effectiveness, for example, to determine if error rates continue to decline over time. We will apply the 'system-related' error classification ${ }^{23}$ to identify system-related error rate and associated mechanisms.

\section{Aim 2}

To assess the effects of the eMM on workflow and efficiency.

\section{Study design and sample}

Observations and interviews will be held with medical, nursing and pharmacy staff at baseline to allow mapping of core work processes associated with medication provision. At each step in the stepped-wedge design, a small number of interviews ( $\sim 4$ on each ward) will be conducted with nursing and medical staff to gain insights into clinical staff perceptions of the impact of the system on workflow, efficiency and care delivery. These interviews will be held 1, 3, 6 weeks and 6 months post-eMM implementation on each study ward. Members of the research team will directly approach hospital staff and 


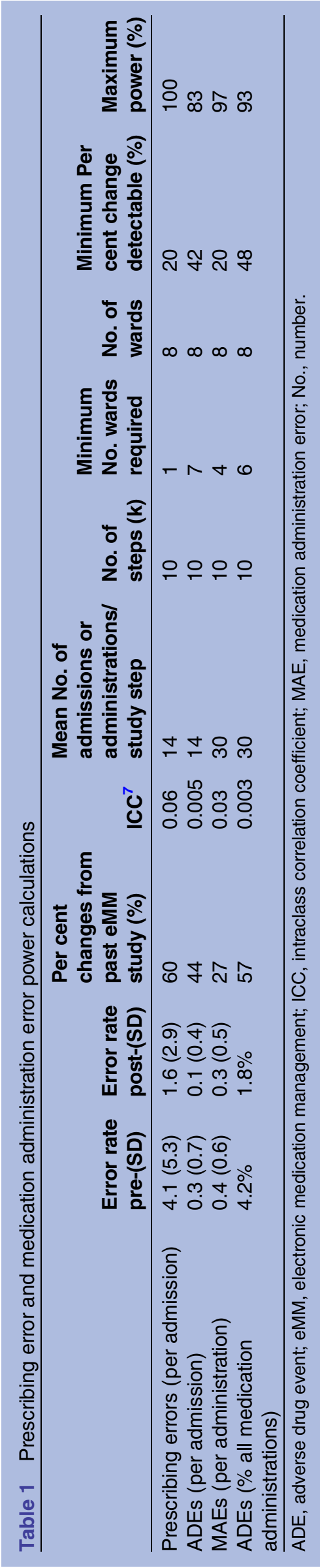

invite them to participate in interviews which will take $\sim 10$ min each. We anticipate $\sim 80-90$ interviews will be conducted across the course of the study. Trustworthiness of the qualitative data will be achieved through triangulation of data and investigators, engagement with the field with a documented audit trail and member checking. ${ }^{34}$

The eMM is anticipated to have a significant impact on the work of hospital pharmacists. We will conduct a direct observational study of $\sim 8$ pharmacists at site 1 . We will observe them for 200 hours between 7:30 and 18:00 pre-eMM and post-eMM system implementation to examine changes in (1) task time distributions, (2) location of work and (3) communication patterns. Using the validated the Work Observation Method By Activity Timing (WOMBAT) approach ${ }^{35}{ }^{36}$ multiple dimensions of work will be captured (eg, tasks performed, with whom, with what, location, interruptions and multitasking). On data entry, tasks are automatically time stamped when entered in the WOMBAT data collection tool. Figure 5 is an example of data collection within WOMBAT. An additional sample of 140 hours of observation will be conducted to capture the work of oncology pharmacists whose work involves supporting the delivery of complex drug regimens to children with cancer.

Data generated will allow changes in task time distributions and sequencing of work to be determined. These data will be examined in relation to changes in outcome indicators generated (from aim 1, eg, medication error rates, LOS) on the same wards.

Aim 3

Assess the extent to which feedback (from aims 1 and 2) and subsequent modifications to an eMM system design can improve eMM system effectiveness in reducing medication errors.

Evaluations of health IT serve multiple purposes, ranging from providing an objective assessment of the success of the new technology in delivering anticipated benefits, to identification of deficits in the system, their source and the ways they can be addressed. This is critical to improving system effectiveness, relevance and responsiveness. For aim 3, the findings of the SWCRCT at CHW (ie, stage 1) will be reported to the Project Evaluation Committee (PEC) made up of members of the research team, and the hospitals' eMM system Project Steering Committee. The PEC will meet every month to consider the implications of study findings across a number of domains including the system's technical features (eg, compatibility with other hospital systems), effectiveness (eg, error reduction and systemrelated errors); professional attitudes (eg, satisfaction) and organisational features (eg, work processes), as a means of formulating changes to eMM system design features and user training.

This will form the key component of an action-oriented approach aimed at optimising system 
Figure 5 WOMBAT for conducting observational studies of health professionals' work pre-eMM and post-eMM system implementation. eMM, electronic medication management; WOMBAT, Work Observation Method By Activity Timing.

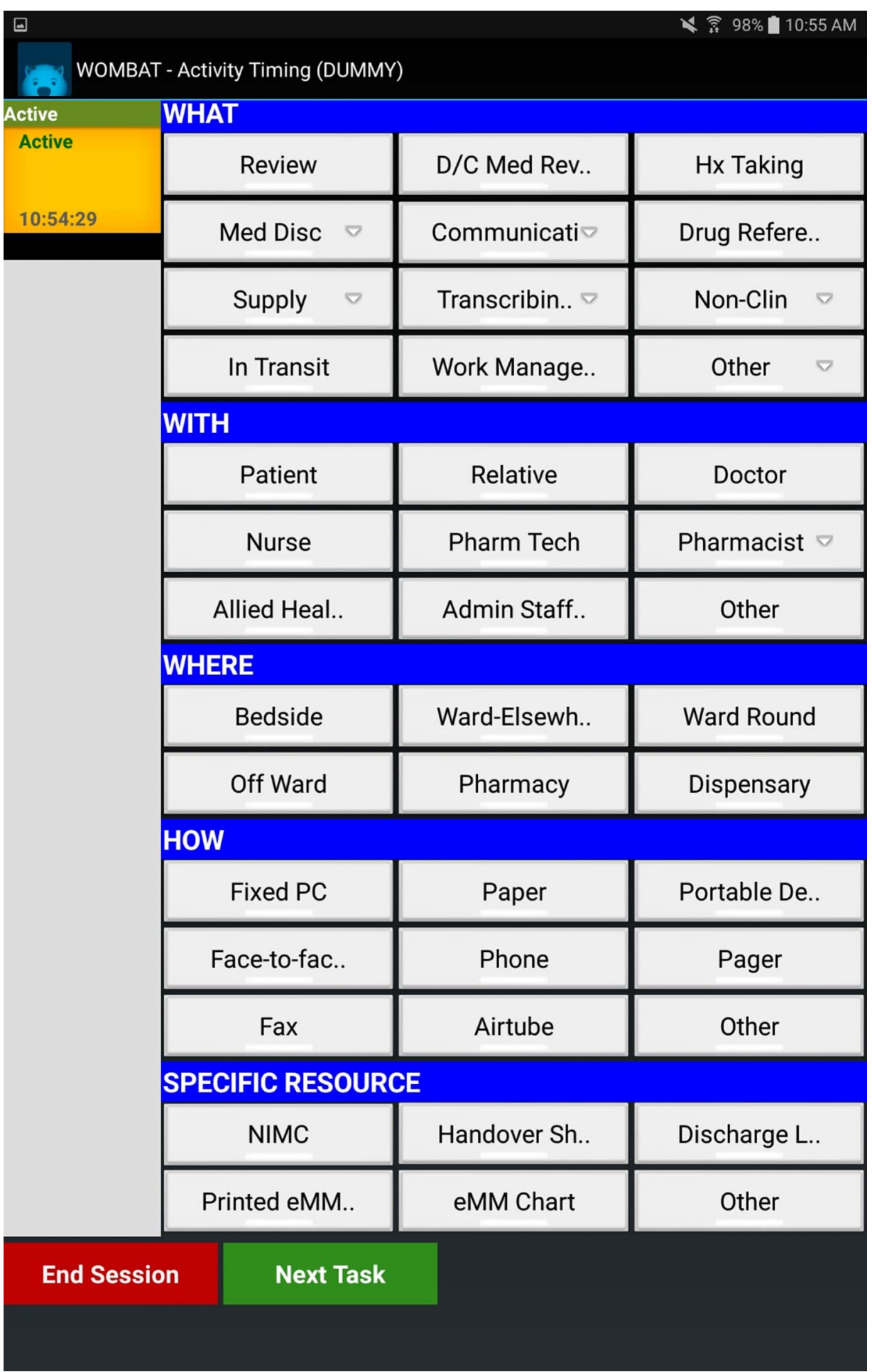

performance leading to an enhanced eMM system which will then be implemented across the second site, SCH (stage 2). The SWCRCT design will be repeated at site 2 using the same methods as above. Power calculations for stage 2 will be based on results from stage 1. We will conduct separate analyses for all outcomes specified. Results for the two sites will be compared, using multilevel and longitudinal analysis approaches to determine changes in error rates (taking baseline data into account).
Expected outcomes and significance of the research project

This project will generate the first Australian data, in a paediatric setting, on the effectiveness of eMM systems to reduce medication errors and ADEs, and provide an assessment of how systems impact on the work of clinicians and the consequences for the delivery of care to children. Importantly, the findings will be directly applied to enhance the eMM system design, and work processes and then tested further through evaluation of 
the enhanced eMM system at a second paediatric hospital. These results will be particularly valuable for other paediatric hospitals yet to start implementations. Exploiting the SWCRCT design within an action research model is highly innovative, and will deliver high-quality data on system effectiveness. Such a model of formally integrating health IT assessment results as a basis for active engagement with IT vendors and clinicians to bring about system change has both national and international significance. The study advances explicit methods for the systematic identification of harm associated with medication errors. The data generated will also provide the basis for a robust cost-effectiveness analysis, which will be the subject of a separate protocol.

\section{ETHICS AND DISSEMINATION}

The research has been approved by the Human Research Ethics Committee of the Sydney Children's Hospitals Network (HREC/15/SCHN/370). In the first instance, results from site 1 will be reported to the PEC so that they can be used to inform eMM system and work process design prior to implementation at site 2. Results will also be reported through academic journals and conference presentations. The project is funded through a National Health and Medical Research Council Partnership Grant. As such, the project team includes academic researchers, hospital clinicians and experts involved in the implementation of the eMM system at the two hospital sites, along with senior policymakers from agencies within the State Health Department involved in eHealth system strategy and policy. This provides the project with access to a range of other conduits through which to disseminate results to, for example, policymakers and system implementers.

\footnotetext{
Author affiliations

${ }^{1}$ Centre for Health Systems and Safety Research, Australian Institute of Health Innovation, Macquarie University, Sydney, New South Wales, Australia

${ }^{2}$ The Sydney Children's Hospitals Network

${ }^{3}$ University of Adelaide

${ }^{4}$ The Sydney Children's Hospitals Network and The University of Sydney

${ }^{5}$ Faculty of Medicine, School of Medical Sciences, University of New South Wales

${ }^{6}$ Vanderbilt University, USA

${ }^{7}$ Office of Kids and Families NSW Health

${ }^{8}$ eHealth NSW Health Ministry
}

Twitter Follow Andrew Georgiou at @AGeorgiouMQ and Johanna Westbrook at @JWestbrook91

Contributors JIW, LL, MTB, AGe and CM initiated the project and led the development of the National Health and Medical Research Council (NHMRC) grant proposal. JIW, LL, MTB, AGe, RD, JK, LD-P, CTC, GA and TAO are chief investigators on the project and all made contributions to the protocol in their specific areas of expertise. CM, LW, AGa, CUL, MG, PK, MK and DB are associate investigators on the NHMRC grant and provided input to the protocol, particularly in the areas of paediatric clinical practice and broader eHealth strategy in relation to eMM systems. MZR, MP, VM, TK, RW, RL are members of the project team and have made significant contributions to the protocol in terms of the design of details regarding the collection and classification of medication errors and harm. JW prepared the first draft of this manuscript based on the grant proposal and all authors have reviewed and provided input.

Funding The project is supported by a NHMRC Partnership Grant (APP1094878) in partnership with: Sydney Children's Hospitals Network; eHealth New South Wales; Office of Kids and Families, New South Wales.

Competing interests None declared.

Ethics approval Human Research Ethics Committee of the Sydney Children's Hospitals Network (HREC/15/SCHN/370)

Provenance and peer review Not commissioned; externally peer reviewed.

Open Access This is an Open Access article distributed in accordance with the Creative Commons Attribution Non Commercial (CC BY-NC 4.0) license, which permits others to distribute, remix, adapt, build upon this work noncommercially, and license their derivative works on different terms, provided the original work is properly cited and the use is non-commercial. See: http:// creativecommons.org/licenses/by-nc/4.0/

\section{REFERENCES}

1. Kaushal R, Barker KN, Bates DW. How can information technology improve patient safety and reduce medication errors in children's health care? Arch Pediatr Adolesc Med 2001;155:1002-7.

2. Doherty C, McDonnell C. Tenfold medication errors: 5 years' experience at a university-affiliated pediatric hospital. Pediatrics 2012;129:916-24

3. Gazarian M, Drew A, Bennett A. Medicinal mishap. Intravenous paracetamol in paediatrics: cause for caution. Aust Prescr 2014;37:24-5

4. Kim GR, Chen AR, Arceci RJ, et al. Error reduction in pediatric chemotherapy: computerized order entry and failure modes and effects analysis. Arch Pediatr Adolesc Med 2006;160:495.

5. Kaushal R, Bates DW, Landrigan $\mathrm{C}$, et al. Medication errors and adverse drug events in pediatric inpatients. J Am Med Assoc 2001;285:2114-20.

6. Miller MR, Robinson KA, Lubomski LH, et al. Medication errors in paediatric care: a systematic review of epidemiology and an evaluation of evidence supporting reduction strategy recommendations. Qual Saf Health Care 2007;16:116-26.

7. Rinke ML, Bundy DG, Velasquez CA, et al. Interventions to reduce pediatric medication errors: a systematic review. Pediatrics 2015;136:583

8. Gazarian M, Graudins LV. Long-term reduction in adverse drug events: an evidence-based improvement model. Pediatrics 2012;129:e1334-e42.

9. Westbrook Jl, Rob MI, Woods A, et al. Errors in the administration of intravenous medications in hospital and the role of correct procedures and nurse experience. BMJ Qual Saf 2011;20:1027-34.

10. Westbrook Jl, Woods A, Rob Ml, et al. Association of interruptions with an increased risk and severity of medication administration errors. Arch Intern Med 2010;170:683-90.

11. Manius E, Kinney S, Cranswick N, et al. Interventions to reduce medication errors in pediatric intensive care. Ann Pharmacother 2014;48:1313-31.

12. Australian Commission on Safety and Quality in Health Care. Electronic Medication management (EMM): a guide for healthcare providers. 2 nd Edition. ACSQHC, Sydney, 2012. https://www. safetyandquality.gov.au/publications/electronic-medicationmanagement-systems-a-guide-to-safe- implementation/

13. van Rosse F, Maat B, Rademaker CMA, et al. The effect of computerized physician order entry on medication prescription errors and clinical outcome in pediatric and intensive care: a systematic review. Pediatrics 2009;123:1184-90.

14. Westbrook JI, Li L, Lehnbom EC, et al. What are incident reports telling us? A comparative study at two Australian hospitals of medication errors identified at audit, detected by staff and reported to an incident system. Int J Qual Health Care 2015;27:1-9.

15. King WJ, Paice N, Rangrej J, et al. The effect of computerized physician order entry on medication errors and adverse drug events in pediatric inpatients. Pediatrics 2003;112(3 Pt 1):506-9.

16. Lehmann CU, Johnson KB, Technology Council on Clinical Information, American Academy of Pediatrics. Electronic prescribing in pediatrics: toward safer and more effective medication management. Pediatrics 2013;131:e1350-e6.

17. Dufendach KR, Eichenberger JA, McPheeters ML, et al. Core functionality in pediatric electronic health records. Agency for Healthcare Research and Quality, 2015. 
18. Slight SP, Berner ES, Galanter W, et al. Meaningful use of electronic health records: experiences from the field and future opportunities. JMIR Med Inform 2015;3:e30.

19. Han YY, Carcillo JA, Venkataraman ST, et al. Unexpected increased mortality after implementation of a commercially sold computerized physician order entry system. Pediatrics 2005;116: 1506-12.

20. Longhurst CA, Parast L, Sandborg Cl, et al. Decrease in hospitalwide mortality rate after implementation of a commercially sold computerized physician order entry system. Pediatrics 2010;126:14-21.

21. Brigham and Women's Hospital, Partners HealthCare, Harvard Medical School. Computerized Prescriber Order Entry Medication Safety (CPOEMS): Uncovering and learning from issues and errors. 2015; https://psnet.ahrq.gov/resources/resource/29442/ computerized-prescriber- order-entry-medication-safety-cpoemsuncovering-and-learning-from-issues-and-errors

22. Westbrook JI, Reckmann M, Li L, et al. Effects of two commercial electronic prescribing systems on prescribing error rates in hospital inpatients: a before and after study. PLoS Med 2012;9: e1001164.

23. Westbrook JI, Baysari MT, Li L, et al. The safety of electronic prescribing: manifestations, mechanisms, and rates of system-related errors associated with two commercial systems in hospitals. J Am Med Inform Assoc 2013;20:1159-67.

24. Koppel R, Lehmann CU. Implications of an emerging EHR monoculture for hospitals and healthcare systems. J Am Med Inform Assoc 2015;22:465-71.

25. McKibbon KA, Lokker C, Handler SM, et al. Enabling medication management through health information technology. Rockville, MD: Agency for Healthcare Research and Quality, 2011.

26. Brown C, Lilford R. The stepped wedge trial design: a systematic review. BMC Med Res Methodol 2006;6:54
27. Westbrook JI, Woods A. Development and testing of an observational method for detecting medication administration errors using information technology. St Heal T 2009;146:429-33.

28. Dean B, Barber N. Validity and reliability of observational methods for studying medication administration errors. Am J Health Syst Pharm 2001;58:54-9.

29. Westbrook JI, Li L, Georgiou A, et al. Impact of an electronic medication management system on hospital doctors' and nurses' work: a controlled pre-post, time and motion study. J Am Med Inform Assoc 2013;20:1150-8.

30. National Coordinating Council for Medication Error Reporting and Prevention. NCC MERP Index for categorizing medication errors 2001 (cited 2015). http://www.nccmerp.org/types-medication-errors

31. NSW Department of Health 2014. Policy Directive-Incident Management Policy, NSW, February 10, 2014; http://www0.health nsw.gov.au/policies/pd/2014/pdf/PD2014_004.pdf

32. Hussey MA, Hughes JP. Design and analysis of stepped wedge cluster randomized trials. Contemp Clin Trials 2007;28:182-91.

33. Lellouche $F$, Mancebo J, Jolliet $\mathrm{P}$, et al. A multicenter randomized trial of computer-driven protocolized weaning from mechanical ventilation. Am J Respir Crit Care 2006:174:894-900.

34. Callen J, Paoloni R, Li J, et al. Perceptions of the effect of information and communication technology on the quality of care delivered in emergency departments: a cross-site qualitative study Ann Emerg Med 2013;61:131-44.

35. Ballerman MA, Shaw NT, Mayes DC, et al. Validation of the Work Observational Method By Activity Timing (WOMBAT) method of conducting time-motion observations in critical care settings: an observational study. BMC Med Inform Decis 2011;11:32.

36. Westbrook Jl, Ampt A. Design, application and testing of the Work Observation Method by Activity Timing (WOMBAT) to measure clinicians' patterns of work and communication. Int $J$ Med Inform 2009;78(Suppl 1):S25-33. 